\title{
Towards identifying malnutrition among infants under 6 months: a mixed- methods study of South-Sudanese refugees in Ethiopia
}

\author{
Laura Moore $^{1}$, Sinead O’Mahony ${ }^{2}$, Mark Shevlin ${ }^{3}$, Philip Hyland ${ }^{1,4}$, Hatty Barthorp ${ }^{2}$, and \\ Frédérique Vallières ${ }^{1,5}$
}

${ }^{1}$ Centre for Global Health, University of Dublin, Trinity College, 7-9 South Leinster Street, Dublin 2, Ireland.

${ }^{2}$ GOAL Global, Carnegie House, Library Road, Dun Laoighre, Co Dublin, Ireland.

${ }^{3}$ Psychology Research Institute, Ulster University, Derry, Northern Ireland.

${ }^{4}$ School of Business, National College of Ireland, Mayor Street, International Financial

Services Centre, Dublin 1, Ireland

${ }^{5}$ School of Psychology, University of Dublin, Trinity College, Ireland.

Corresponding author: Laura Moore, 1moore2@tcd.ie, +64276195593.

Short Title: Identifying malnutrition in infants under 6 months

Acknowledgements: We thank the US State Department's Bureau of Population, Refugees and Migration (BPRM), ECHO, UNICEF and UNHCR who funded the "Emergency Nutrition Response for South Sudanese refugees in Gambella Region, Ethiopia', within which the 'Community Management of At Risk Mothers and Infants under 6 months (CMAMI)' project was nested.

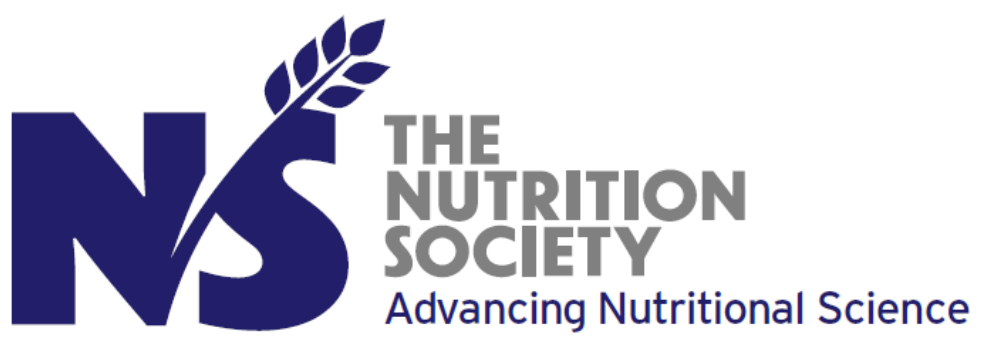

This is an Accepted Manuscript for Public Health Nutrition as part of the Cambridge Coronavirus Collection. This peer-reviewed article has been accepted for publication but not yet copyedited or typeset, and so may be subject to change during the production process. The article is considered published and may be cited using its DOI $10.1017 / \mathrm{S} 1368980020004048$

Public Health Nutrition is published by Cambridge University Press on behalf of The Nutrition Society 
Financial Support: This research received no specific grant from any funding agency, commercial or not-for-profit sectors

\section{Conflict of Interest: None}

\section{Authorship:}

Laura Moore- Primary researcher and author of manuscript, study design, literature review, collected qualitative data, data analysis, formation of results and conclusions. Sinead O'Mahony- Link to GOAL, design key informant interview template, provided information regarding secondary data, reviewed and edited manuscript. Mark Shevlin- Statistical analysis, formation of quantitative results . Philip Hyland- Statistical analysis, formation of quantitative results. Hatty Barthop- Link to GOAL, reviewed and edited manuscript. Frédérique Vallières- Reviewed and edited manuscript, provided academic feedback throughout research and development of manuscript, study design, quantitative results and discussion.

Ethical Standards Disclosure: Approval to access the de-identified secondary data was obtained from GOAL. GOAL also acted as a gatekeeper, linking the Lead Author to the MAMI special interest group. Potential informants were initially invited to participate by email and both verbal and written consent were obtained prior to the start of the interview. Ethical approval to perform the study was obtained by HPM/Centre for Global Health Ethics Committee, Trinity College Dublin. 


\begin{abstract}
Objectives: To determine (i) whether distinct groups of infants under 6 months old (U6Ms) were identifiable as malnourished based on anthropometric measures and if so, to determine the probability of admittance to GOAL Ethiopia's Management of At Risk Mothers and Infants (MAMI) programme based on group membership; (ii) whether there were discrepancies in admission using recognised anthropometric criteria, compared to group membership, and (iii) the barriers, and potential solutions, to identifying malnutrition within U6Ms.
\end{abstract}

Design: Mixed methods approaches where used, whereby data collected by GOAL Ethiopia underwent: factor mixture modelling, chi-square analysis and logistic regression analysis. Qualitative analysis was performed through coding of key informant interviews.

Setting: Data were collected in two refugee camps in Ethiopia. Key informant interviews were conducted remotely with international MAMI programmers and nutrition experts. Participants: Participants were 3,444 South-Sudanese U6Ms and 11 key informants experienced in MAMI programming.

Results: Well-nourished and malnourished groups were identified, with notable discrepancies between group membership and MAMI programme admittance. Despite weight for age z-scores (WAZ) emerging as the most discriminant measure to identify malnutrition, admittance was most strongly associated with mid-upper arm circumference (MUAC). Misconceptions surrounding malnutrition, a dearth of evidence, and issues with the current identification protocol emerged as barriers to identifying malnutrition among U6Ms.

Conclusions: Our model suggests that WAZ is the most discriminating anthropometric measure for malnutrition in this population. However, the challenges of using WAZ should be weighed up against the more scalable, but potentially overly sensitive and less accurate use of MUAC among U6Ms.

Key Words: Infants under 6 months, Malnutrition, MUAC, WAZ, WLZ, MAMI. 


\section{Abbreviations}

AM - Acute Malnutrition

EBF - Exclusive Breastfeeding

HCW - Health Care Workers

IYCF - Infant and Young Child Feeding

KIIs - Key Informant Interviews

MAMI - Management of At Risk Mothers and Infants

MUAC - Mid-upper Arm Circumference

U6Ms - Infants Under 6 Months of Age

WAZ - Weight for Age z-score

WHO - World Health Organization

WLZ - Weight for Length z-score 


\section{Introduction}

A primary contributor to the global burden of disease ${ }^{(1)}$, malnutrition accounts for $53 \%$ of under-5 mortality ${ }^{(2)}$. Large-scale efforts to assess the outcomes of children enrolled in nutrition programmes have identified higher rates of acute malnutrition (AM) or wasting, the result of recent rapid weight loss or the failure to gain weight ${ }^{(3)}$, among infants under the age of 6 months (U6Ms), compared to those aged from 6-59 months ${ }^{(4)}$. This represents an increased vulnerability for malnutrition among U6Ms, which, if not addressed, could result in severe and irreversible adverse health outcomes.

A number of inter-connected factors contribute to the increased vulnerability of U6Ms. Firstly, U6Ms have unique physiology ${ }^{(5)}$ including active immune suppression in early infancy ${ }^{(6)}$ which can correspond to increased risk, frequency, duration and severity of infection $^{(3)}$. The immune system response during nutrient shortage and adipose tissues role in nutrient uptake are interrelated processes ${ }^{(7)}$. Furthermore, infections may lead to an increased demand for calories exacerbating malnutrition ${ }^{(8)}$. These interrelated factors can create a cycle of malnutrition and worsening illness ${ }^{(3)}$.

Secondly, considering U6M's complete dependence on caregivers' decisions regarding feeding practices ${ }^{(9)}$, certain cultural beliefs can contribute to malnutrition. Restrictive practices around breastfeeding, food taboos, and misconceptions that the colostrum is dangerous or 'dirty' for infants, have been identified as important global determinants of infant malnutrition ${ }^{(10,11)}$. While international efforts to curb child malnutrition have emphasised the importance of exclusive breastfeeding (EBF), U6Ms have long been considered less vulnerable to malnutrition due to the assumption that infants are exclusively breastfed. However, infants U6M are vulnerable to AM regardless of breastfeeding status ${ }^{(4)}$. Moreover, and although effective EBF can protect against early malnutrition, it is only practiced with an estimated $40 \%$ of all U6Ms ${ }^{(12)}$. Consequently, millions of infants are exposed to contaminated water, pre-lacteals and inappropriate foods annually, potentially causing illness and further malnutrition ${ }^{(13,14)}$.

Due to these misconceptions, infants are commonly overlooked in community screenings for malnutrition and standard nutrition surveys ${ }^{(15)}$ and are often excluded from nutritional recommendations and interventions ${ }^{(4)}$. Often programmes for the identification and treatment of AM in "infants and children" refer exclusively to those 6-59 months ${ }^{(16)}$. Furthermore, it has been found that even when admitted to therapeutic feeding programmes U6Ms had more missing anthropometric data than their 6-60 months counterparts ${ }^{(17)}$. 
As a preventative strategy, infant and young child feeding (IYCF) practices have the greatest potential to improve child survival ${ }^{(18)}$. The potential of IYCF has been acknowledged by the international community through numerous inter-sectoral campaigns focussing exclusively on nutrition including The Decade ${ }^{(19)}$ and The Scaling Up Nutrition Movement ${ }^{(20)}$. Here again, however, there is a conspicuous absence of U6Ms within these campaigns and programmes. Taken together, the above not only demonstrates that U6Ms are at a greater risk of malnutrition compared to their older counterparts, but they are also indirectly at risk, due to the widespread exclusion of this age group from current child malnutrition literature, policy, and diagnostic guidelines. This dearth of evidence has a cascading effect, with policy makers reluctant to ratify policies to identify and treat AM in U6Ms without substantial rationale. Subsequently, practitioners and humanitarian workers face a notable lack of guidance when they encounter infants U6M whom appear to be malnourished. Furthermore, despite the World Health Organisation (WHO) stating outpatient care should be available for U6M with AM this is not reflected in in national protocols, thus leaving inpatient treatment as the only option for high risk cases ${ }^{(21)}$. There are also concerns that inaccurate assessments amongst small but healthy infants could be counterproductive by undermining and/or interrupting $\mathrm{EBF}^{(4,22)}$.

Unfortunately, the paucity of malnutrition research conducted among U6Ms has resulted in a dearth of guidelines and protocols for how to best measure and identify AM for this age group ${ }^{(23)}$. As a result, at the time of data collection there was an overreliance on tools and methods developed and tested for children 6-59 months being applied to the identification of AM for U6Ms. Among children 6-59 months, AM is most often measured and identified using weight-for-age (WAZ), weight-for-height (WHZ) or weight-for-length (WLZ) Z-scores and/or a measure of mid-upper arm circumference (MUAC) ${ }^{(24)}$. However, inconsistencies between these tools are well documented within the literature ${ }^{(25)}$. Given the availability of different anthropometric indicators of malnutrition (i.e., WLZ, WAZ, MUAC), and the absence of evidence regarding which of these is best to assess malnutrition among U6Ms, an alternative approach may be to determine if there are unique groups of U6Ms characterised by similar patterns of malnutrition across these different measures.

\section{Aims and Objectives}

The identification of malnutrition within U6Ms is necessary to mitigate the physiological, socio-cultural and political factors which can contribute to an increased risk of malnourishment within this population. We conducted a study of infants aged 0-6 months 
residing in two refugee camps in Ethiopia. Our objectives were to determine; (i) whether distinct groups of malnourished U6Ms were identifiable based on multiple anthropometric measures; and if so, to determine the probability of admittance to GOAL Ethiopia's Management of At Risk Mothers and Infants (MAMI) programme based on one's group membership; (ii) whether there were discrepancies in admission to MAMI, using anthropometric criteria internationally recognised at the time of data collection as best practice (i.e. MUAC < 110mm, MUAC < 115mm, WLZ <-3.0, WAZ < -3.0), compared to admission based on group membership; (iii) the key barriers, and possible solutions, to the challenge of identification of malnutrition within U6Ms, from the perspective of global child nutrition experts.

\section{Methods}

\section{Participants and Procedures}

The first two objectives used data collected by GOAL Ethiopia's MAMI programme as part of the routine, monthly programme collection. Screening was linked to blanket supplementary feeding distribution and maternal, infant and young child nutrition education as part of a wider nutrition support programme run in collaboration with the United Nations High Commissioner for Refugees and the Ethiopian Administration for Refugee and Returnee Affairs. At the time of commencing this study there were data on 3,444 infants U6Ms. Thus, participants were 3,444 South-Sudanese U6Ms (51.9\% female, mean age $=2.98$ months, $\mathrm{SD}=1.34$ ) seeking refuge in Ethiopia and residing in either Kule or Tierkidi camps. The data were collected between $22^{\text {nd }}$ February 2016 and $2^{\text {nd }}$ January 2017.

The third objective made use of non-probability, snowball sampling procedures to recruit key informants with extensive experience with MAMI implementation and programming ${ }^{(26)}$. The key informant interviews (KIIs) consisted of nine females and two males, all over 18 years of age. Participants were recruited from multiple humanitarian and academic organisations including: Action Against Hunger, Save the Children UK and US, Brixton Health, the WHO, the Directorate-General for European Civil Protection and Humanitarian Aid Operations (ECHO) and the London School of Hygiene and Tropical Medicine. All interviews were conducted by the lead author in English over Skype and lasted an average of 24.7 minutes. Written informed consent was gained prior to the interviews. 


\section{Measurement Tools}

Weight was measured using $25 \mathrm{~kg}$ salter scales with $100 \mathrm{~g}$ increments, while length was measured using a solid height board laid flat on the ground. MUAC measurements were taken using a standard UNICEF specification colour coded MUAC tape. Mothers/carers were asked age of the infant in months. All data were collected in a clinic setting by trained nurses or health extension agents on paper forms and entered into a database by the nutrition programme manager. Consistent with international guidelines ${ }^{(21,27)}$, the following cut-offs for severe acute malnutrition were employed to assess malnutrition in U6Ms: (i) WLZ $<-3.0$ and (ii) WAZ $<-3.0$. In addition, (iii) MUAC $<110 \mathrm{~mm}$ and (iv) $<115 \mathrm{~mm}$ were utilised based on the cut-offs for children 6-59 months ${ }^{(21,28)}$ as there is currently no internationally recognised MUAC cut-off for U6M. In line with these internationally recognised definitions, GOAL's MAMI protocol specified that admission at the time should be based on anthropometric measurements of MUAC $<115 \mathrm{~mm}$ and $\mathrm{WLZ}<-2$, also recent weight loss, failure to gain weight or visible wasting. This was decided by nursing staff, supported by community outreach agents. These decisional factors, outside anthropometry, were not recorded in the data set.

The interview schedule was designed based on the above review of the literature, with input from GOAL's nutritional advisor. The final schedule included open-ended questions designed to solicit responses and opinions on the factors that facilitate and prohibit the identification of malnutrition in U6M (see Appendix 1). The schedule was piloted with a nutrition expert prior to use, leading to minor changes to clarify the language in the interview guide. The lead author performed the interviews. These were audio recorded and transcribed verbatim immediately following each interview. Following this, the transcriptions were crosschecked with the audios for accuracy and for additional notes.

\section{Data Analysis}

Z-scores in the dataset were recorded using look-up tables. Factor mixture modelling was used to determine whether empirically distinct groups of U6Ms were identifiable within this population based on their scores across three anthropometric measures (WAZ scores, WLZ scores, and MUAC z-scores ${ }^{1}$ ). Factor mixture modelling is a latent variable modelling technique that combines confirmatory factor analysis (CFA) with latent profile analysis

\footnotetext{
${ }^{1}$ MUAC scores were transformed to z-scores for the purposes of Factor Mixture Modelling so that all measures were equally scaled.
} 
(LPA) in a simultaneous process ${ }^{(29)}$. First, the associations between scores on the three anthropometric measures were modelled as observed indicators of a single latent dimension of malnutrition (CFA component). Second, variation in levels of nutrition were held constant and the optimal number of groups (or 'latent classes') of U6Ms were determined (LPA component). LPA is well-suited to this study as it is an exploratory, data-driven technique that makes no a priori assumptions regarding the number of groups that exist in the population $^{(30)}$

Four factor mixture models were tested that included one factor (nutrition) and one to four latent classes. All models were estimated using the robust maximum likelihood estimator (31) and missing data was managed using full information maximum likelihood. To avoid solutions based on local maxima, 500 random sets of starting values were used, followed by 50 final stage optimizations. The relative fit of the models were compared using three information theory based fit statistics: the Akaike Information Criterion ${ }^{(32)}$, the Bayesian Information Criterion ${ }^{(33)}$ and the sample size adjusted Bayesian Information Criterion ${ }^{(34)}$. In each case the model with the lowest value is considered to be the best, and Nylund, Asparouhov and Muthen ${ }^{(35)}$ demonstrated that the BIC is the best information criterion for identifying the correct number of classes. In addition, the Lo-Mendell-Rubin adjusted likelihood ratio test ${ }^{(36)}$ was used to compare models with increasing numbers of latent classes. A non-significant value ( $\mathrm{p}>.05)$ suggests that the model with one less class should be accepted. These analyses were conducted using Mplus 7.11 ${ }^{(37)}$.

Next, bivariate associations between group membership and the likelihood of MAMI admission, as well as WHO cut-offs for malnourishment and likelihood of MAMI admission, were assessed using chi-square analysis. Group membership was defined by the results of the factor-mixture model. Given high rates of missing data on the MAMI admission variable, differences between U6Ms who had no information on admittance (i.e. missing cases), those admitted, and those who were not admitted for MAMI on measures of MUAC, WAZ, and WLZ were assessed using a one-way between groups analysis of variance (ANOVA). Logistic regression analysis was conducted to determine the associations between each anthropometric measures and admittance for MAMI, controlling for sex $(0=$ male, $1=$ female) and age.

The challenges with identifying malnutrition within U6Ms, as perceived by global child malnutrition experts, were identified through key informant interview. Interviews were transcribed verbatim immediately following each interview and transcriptions were crosschecked with the audios for accuracy and for additional notes (i.e. pauses). Data was 
analyzed using inductive approaches, via open coding. Generated codes were then categorized through axial coding whereby original codes were subsumed under broader categories ${ }^{(38)}$. Finally, selective coding was applied to identify key emergent themes in the creation of key concepts ${ }^{(38)}$

\section{Results}

\section{Descriptive statistics}

From the total sample of 3,444 U6Ms $(51.9 \%$ female, mean age $=2.98$ months, $\mathrm{SD}=$ 1.34), 5.9\% $(\mathrm{n}=202)$ were admitted to GOAL Ethiopia's MAMI programme, 59.2\% were not admitted and $34.9 \%$ had no record of whether they were admitted or not admitted. With respect to the different anthropometric measures of malnutrition, 8.3\% $(\mathrm{n}=286)$ of U6Ms met the criteria for malnutrition based on MUAC scores $<115 \mathrm{~mm} ; 3.7 \%(\mathrm{n}=129)$ based on MUAC scores $<110 \mathrm{~mm} ; 5.1 \%(\mathrm{n}=177)$ based on WAZ scores $<-3.0$; and 3.9\% $(\mathrm{n}=133)$ based on WLZ scores $<-3.0$ (see Table 1).

\section{Missing Data Analysis}

Significant differences between those with missing data on admittance, those who were admitted, and those who were not admitted were identified across all three anthropometric measures $(p s<0.001)$. Post-hoc analysis suggested no differences between U6Ms who had no information on admittance and those not admitted to MAMI. Differences were found, however, on those who were admitted for MAMI, whereby those who were admitted were recorded as having lower WAZ, WLZ, and MUAC scores. This suggests that the reason why no record of admittance was made in certain cases is possibly due to the absence of the U6M meeting any of the cut-off criteria for malnutrition.

\section{Objective 1}

The factor mixture modelling results identified two distinct groups of U6Ms in this population (see Table 2). The first group included $94.3 \%(n=3,248)$ of U6Ms, with infants in this group characterised by normative scores on each anthropometric measures of nutrition $(\mathrm{WAZ}=-0.10, \mathrm{WLZ}=-0.22$, and mean MUAC $=132.29)$. The second group included $5.7 \%$ $(n=196)$ of U6Ms, and these infants were characterised by WAZ scores of -3.86 , WLZ scores of -1.61 , and mean MUAC scores of $114.80 \mathrm{~mm}$; thus, reflecting a group of U6Ms 
experiencing malnutrition (see Table 3). The profile plots for both groups are represented in Figure 1.

\section{TABLES 1,2 \& 3 HERE}

The association between group membership and admission for MAMI was statistically significant $(\chi 2=226.39, \mathrm{df}=1, \mathrm{p}<.001)$, whereby individuals in the malnourished group were 10.79 times more likely to be admitted for therapeutic intervention than those in the well-nourished group $(\mathrm{OR}=10.79,95 \% \mathrm{CI}=7.47-15.60)$. Only $43.2 \%$ of U6Ms in the malnourished group were admitted for MAMI; and of all U6Ms who were admitted for MAMI, only $31.7 \%$ belonged to the malnourished group.

\section{FIGURE 1 HERE}

\section{Objective 2}

The results of the bivariate and multivariate associations between admittance for MAMI and meeting internationally recognised criteria for AM are presented in Table 4. Admittance to MAMI was most strongly associated with MUAC scores $<115 \mathrm{~mm}\left(\mathrm{OR}_{\mathrm{adj}}=\right.$ 366.03), followed by WLZ scores $<-3.0\left(\mathrm{OR}_{\mathrm{adj}}=2.89\right)$, and WAZ scores $<-3.0\left(\mathrm{OR}_{\mathrm{adj}}=\right.$ 2.12). Cross-tabulations indicated that $82.9 \%$ of U6Ms with MUAC scores $<115 \mathrm{~mm}$ were admitted for MAMI, 30.7\% of U6Ms with WAZ scores < -3.0 were admitted for MAMI, and $26.7 \%$ of U6Ms with WLZ scores $<-3.0$ were admitted for MAMI.

\section{TABLE 4 HERE}

\section{Objective 3}

A number of key themes emerged with regards to barriers to the identification of AM within U6Ms: (i) HCW misconceptions surrounding the existence and cause of AM in this age group, (ii) a dearth of evidence, and (iii) issues with the current identification protocol, especially with measures of WLZ. Misconceptions regarding the nature of AM in U6M described as a "stigma" (P1) and one of the "biggest hindrances" (P4) was mentioned frequently ( $n=7)$ and in length. Namely, the lack of awareness and recognition that AM can and does exist in U6Ms was seen as an important barrier, as explained by Participant 4: "The biggest challenge has been the assumption that there is no malnutrition within this age group... because then if you don't think there is a problem you don't look for it". Misconceptions further included the belief among fieldworkers that AM is "impossible" in $\mathrm{U} 6 \mathrm{M}(\mathrm{n}=3)$, with some rationalising this with the fallacy that U6Ms are protected from AM due to $\mathrm{EBF}(\mathrm{n}=2)$. It was suggested that this lack of awareness contributes to a lack of effort to identify AM among U6Ms (P11). 
Ubiquitous across each interview was the second theme of a lack of evidence regarding the identification of AM in U6M. Described by P8 as the "black hole in terms of data", with standard surveys focusing on 6-59 months, a systematic exclusion of U6M infants $(n=3)$ has led to a lack of longitudinal data on MUAC for U6M (P4). As explained by Participant 2: "The number of AM children U6M is not a figure or a measure that we see very often in reports, so people have a tendency, I think, of thinking that it's quite rare and therefore it's never something that is necessarily prioritised". With insufficient research on the issue, one is unable to demonstrate need and consequently incite adequate action $(n=2)$. It was proposed that the systematic measurement of MUAC for this age group will, in itself, generate data (P5).

All 11 interviewees mentioned the current WLZ case definition as a barrier to identification of AM in U6Ms, with the criteria being described as "very rigid" and "legalistic" (P3). Issues with WLZ arose at each stage of the WLZ process. Firstly, obtaining the anthropometric measurements of weight and length were considered difficult due to the poor availability of equipment $(n=2)$, with procurement of equipment being a particularly big barrier in rural settings (P9). The reliability of the equipment was also mentioned in terms of the accuracy of weighing scales $(n=2)$, and the difficulty of measuring the physicality of U6Ms equating to a larger margin of error in terms of measurement (P2). Where equipment was available, the physical measurement itself can be inconsistent, i.e. removal of infant's clothes before weighing (P10), with difficulties obtaining U6M height being described as "particularly challenging" (P2), and requiring more resources, including more staff, "at least 2 people, ideally 3 , whom are a higher capacity of health worker" (P5) and time (n=2), whereby "realistically people won't have time to do it in practice in the field, it won't be done". Finally, the majority of participants expressed that even when WLZ is performed well it is not an ideal measurement for U6Ms ( $n=9)$. Issues with WLZ being "not necessarily reliable or accurate" (P4) for U6Ms included WLZ being superimposed from the 6-59month age group without much supporting evidence (P4), and not accounting for low birth weight infants or those small for gestational age $(n=2)$.

Although considered a "very poor prognostic indicator of mortality" (P3), the transition from WLZ to another measure was flagged as difficult: "I think it's fetishized in a way...people really, really stick to this (WLZ)...no matter how much evidence you give to people they will still go out and use weight for height" (P3). There appeared to be a further barrier in the way of overcoming this, as other feasible options such as MUAC are not 
supported with official guidance and thus its use is prevented $(n=2)$, one participant even considered this the "biggest barrier" to the identification of AM in U6Ms (P5).

To address these barriers, a number of possible solutions were acknowledged, including using a more appropriate tool for identification, whereby the majority of participants named MUAC as the preferred option. MUAC was described as "the only way forward really" (P1), with its perceived advantages being its simplicity, efficiency, low cost and ease of transport and use $(\mathrm{n}=2)$, in addition to possibly increasing access to screening and increasing the number of detected cases $(n=2)$. As participant 11 stated: "I think the evidence is becoming stronger and stronger that MUAC is the way forward for this group as, and will, is the one thing that will revolutionise their management". Furthermore, MUAC and WAZ were flagged as more practical (P1), easier to use (P10), and better predictors of mortality (P4) in comparison to WLZ. However, all of those that did explicitly mention MUAC discussed the needed to use appropriate cut-offs $(n=6)$.

Although breastfeeding was mentioned by 10 of the 11 participants, it was expressed as both a potential facilitator $(n=4)$ and barrier $(n=6)$ to the identification of AM in U6M. Breastfeeding emerged as a facilitator to identification of AM, particularly where there is a lack of equipment for WLZ measures, "the only thing that they can do is assess if there is any breastfeeding problem... so they need to be trained on that simple, simple rapid assessment of breastfeeding problem". Health care workers (HCW) available and already trained to assess breastfeeding practices in combination with clinical signs of AM were also perceived to be facilitators to the identification of AM in U6M $(n=4)$. Numerous interviewees expressed that they felt breastfeeding assessment should be used for identification in collaboration with anthropometry ( $n=3)$, while some suggested the case definition of AM in U6M should include risk factors such as breastfeeding practices $(\mathrm{n}=2)$. 
Breastfeeding was also portrayed as a potential barrier to the identification of AM. As participant 11 explained: "I think there's that false logic that infants should be breastfed and therefore malnutrition is rare in that age group".

The assumption that $\mathrm{U} 6 \mathrm{M}$ are exclusively breastfed and thus are protected from AM was described as a key barrier $(n=2)$, breastfeeding and IYCF practices are an additional challenge particular to this cohort of children (P10). Subsequent treatment related to breastfeeding following the diagnosis of AM was also suggested to be a barrier to identification as it disincentivized $\mathrm{HCW}$ :

Identification would kind of have to be accompanied by treatment and because with infants under 6months that would have to be a lot about breastfeeding and kind of counselling the mother and kind of supporting the mother ... that's also a kind of barrier for even starting the identification because of the treatment that's implied (P10)

Finally, the lack of evidence of breastfeeding benefits for rehabilitating malnourished infants was suggested to further disincentivize $\mathrm{HCW}$ to perform the initial identification (P4), with the idea of treating a malnourished infant U6M with anything other than the breast being described as "taboo" (P3). It was noted that a "kneejerk reaction" by influential breastfeeding advocates, referred to by one participant as "breast fundamentalists" could potentially hinder progress in the identification of AM in U6Ms.

\section{Discussion}

U6Ms are routinely excluded from malnutrition prevalence rates and nutrition interventions globally, prompting MAMI experts and a number of international agencies to call for a stronger evidence base for efficient management of AM in infants ${ }^{(21,39,40)}$. Part of this is due to misconceptions regarding the existence and causes of malnourishment in U6M, including the assumption that U6M are inherently protected from malnourishment by breastfeeding. There is some overlap between our KII findings, regarding perceived causes of AM in U6Ms and breastfeeding practices, and similar KIIs within the literature ${ }^{(41)}$. In light of 
the current absence of an agreed upon method by which to most effectively identify malnutrition among U6Ms, we applied novel statistical methods (factor mixture modelling) that used information from WAZ, WLZ, and MUAC scores to determine whether there were two distinct groups within this population. One group, representing $94.3 \%$ of the population, were characterised by normative WAZ $(-0.10)$ and WLZ $(-0.22)$ scores, and a mean MUAC score of $132.29 \mathrm{~mm}$. These anthropometric measures are consistent with international guidelines for healthy levels of nourishment. The second group, representing the remaining $5.7 \%$ of the population, were characterised by extremely low WAZ scores $(-3.86)$, low WLZ scores (-1.58), and a mean MUAC score of 114.80mm. Consistent with recognised cut-off criteria for 6-59months, including WAZ of $<-3.0$ and for MUAC scores of $<115 \mathrm{~mm}$, as well as others who found non-negligible levels of wasting among U6Ms ${ }^{(4)}$, we therefore found evidence of the existence of malnourishment among this age group. Further, and also consistent with previous findings ${ }^{(42)}$, our results suggest that malnourished children can be most effectively differentiated from their nourished counterparts by the WAZ anthropometric measure. Our results also support the use of MUAC $<115 \mathrm{~mm}$ as an appropriate cut-off to identify malnutrition in this population; a finding that is consistent with the most recent revisions made to MUAC cut-offs by the $\mathrm{WHO}^{(21)}$.

Among the total population of U6Ms, 5.9\% were recorded as having been admitted for MAMI. Our findings suggest the possibility that a large number of U6Ms belonging to the well-nourished group were admitted for MAMI. Conversely, more than half of U6Ms belonging to the malnourished group were excluded from MAMI programming. These results highlight the possible consequences of the lack of availability of clear protocols and guidelines around the identification and admission criteria for U6Ms, a theme which also emerged as a perceived barrier to the identification of AM in U6M within the qualitative interviews. Specifically, the absence of evidence-based guidelines will inevitably result in 
the misallocation of scarce resources, the exclusion of vulnerable U6Ms from life-saving interventions, and the unnecessary inclusion of U6Ms and their caretakers in what amounts to time and resource intensive therapeutic interventions.

In relation to the study's second objective, results of the bivariate and multivariate analysis indicated that admittance to MAMI, in practice, was most strongly associated with use of the MUAC criterion of scores $<115 \mathrm{~mm}$. Although amongst the literature MUAC is recognised as a reliable measure among children 6-59 months valued for its simplicity, accuracy, reproducibility, and affordability ${ }^{(25,43,44)}$ it has also been criticised for its strong association with gender, age and stunting therefore negatively affecting its validity ${ }^{(25)}$. Consistent with internationally recognised guidelines, U6Ms that met this criterion (MUAC $<115 \mathrm{~mm}$ ) were 366 times more likely to be admitted than those that did not, controlling for sex, age, and all other anthropometric measures. In contrast, despite WAZ emerging as the most discriminating anthropometric measure, approximately $70 \%$ of U6Ms that satisfy this criterion (WAZ <-3.0) are not being admitted for treatment for malnutrition.

There is therefore a clear disconnect between the anthropometric measure that most clearly distinguishes U6Ms in the malnourished group and those in the well-nourished groups (i.e. WAZ scores), and the anthropometric measure that was most strongly associated with MAMI admission (i.e. MUAC scores $<115 \mathrm{~mm}$ ). As gleaned from the qualitative interviews however, this is likely due to the simplicity, affordability and ease of use of the MUAC over more onerous and time-consuming methods, where equipment (i.e. weighing scales) is not always available or reliable ${ }^{(25,43)}$. Therefore, while a greater reliance on WAZ scores for the identification of malnutrition should lead to improved access to treatment for those U6Ms most in need of care, this needs to be considered in light of the practicality of other, more sensitive methods. Furthermore, although this study explored admittance to MAMI not mortality, among 1- 6 month olds, Mwangome et al. ${ }^{(42)}$ found that WAZ and MUAC were 
better predictors of mortality than WLZ. Consistent with others suggesting the best cut-off for MUAC to be $<110 \mathrm{~mm}^{(21,42)}$, Mwangome et al. ${ }^{(42)}$ found this to be a greater predictor of mortality among 1-6 month olds than WLZ scores <-3.0.

A stronger emphasis on the use of WAZ is consistent with international guidelines of growth monitoring among children under-2 years of age. However, the challenge remains in weighing the additional financial and human resource costs of using WAZ against the more scalable, but potentially overly sensitive and less accurate, use of MUAC scores. Aligned with the results of the factor mixture modelling, interviewed international nutrition experts identified WAZ and MUAC scores as the most practical methods to identify malnutrition, compared to WLZ. Unanimously WLZ was reported as a barrier to the identification of AM by MAMI experts, concerns consistent with the literature such as the exclusion of smaller infants $<45 \mathrm{~cm}$ from WLZ plotting ${ }^{(45,17)}$ were noted.

Furthermore, the efficiency of MUAC in the field over WLZ based on the reliability of measurements taken by $\mathrm{HCW}$ is also reflected within the literature ${ }^{(43)}$. As noted by Kerac et al. in addition to the reliability of WLZ being questionable there is limited evidence of its validity and accuracy in U6Ms ${ }^{(46)}$. Practically, WLZ is a less favoured indicator given the challenges associated with measuring the length of an infant. Consequently, and as Grijalva-Eternod et al (2017) note, length is often missing in admission data for U6Ms ${ }^{(17)}$. Specifically, in terms of practical use, it was found that procurement and reliability of equipment for measuring weight/height and inconsistencies during the measurement process are perceived barriers to the identification of malnutrition. This is concurrent with literature detailing barriers such as fears of harming the infant, unfamiliarity with taking weight and height measurements for such a young age group and the use of differing scales ${ }^{(4)}$ noted as negative aspects to the standard anthropometric measurements. 
Limitations: The current study is not without limitations. First, this being a cross-sectional study, there is an absence of outcome criteria (i.e. infant mortality) with which to compare the different anthropometric criteria. As such, we cannot infer which anthropometric criteria is most effective to predict mortality. Similarly, the absence of confounding factors such as maternal age and education, number of other children in the household, and the presence of disease or pitting oedema among infants, as additional factors that could have influenced programme admittance, is another limitation of this study. Third, as the data were manually transcribed from paper forms to the data base there are potential for transcription errors. Fourth, as the sample were U6M refugees, whom, as discussed, present a particular vulnerability to malnutrition, the findings may not be generalisable to other infants U6M.

\section{Conclusions:}

The lack of a standardised identification tool for malnutrition in U6M potentially equates to the exclusion of many vulnerable U6Ms from potentially life-saving MAMI programmes while a proportion of healthy U6Ms are unnecessarily enrolled for resource intensive inpatient therapeutic interventions. This study shows a clear disconnect between the most discriminant anthropometric measurement WAZ, and the anthropometric measurement with the most associated admissions to therapeutic intervention (WLZ). The statistically driven results are consistent with expert opinions that MUAC is a preferred method of anthropometric measurement to identify malnutrition in U6Ms in the field. However, the scalability, ease of use and reduced human and time resources associated with MUAC needs to be considered against the specificity and reliability of WAZ. Further research is required for future predictions of morbidity and mortality outcomes based on the use of the different anthropometric measurements. 


\section{References}

1. International Food Policy Research Institute (IFPRI) (2016) Global Nutrition Report 2016: From Promise to Impact: Ending Malnutrition by 2030. Washington: IFPRI.

2. Fekadu Y, Mesfin A, Haile D et al. (2015) Factors associated with nutritional status of infants and young children in Somali Region, Ethiopia: a cross- sectional study. BMC Public Health 15(846), 1-9.

3. United Nations Children's Fund (UNICEF) (2018) Nutrition: Malnutrition: Current Status and Progress. http://data.unicef.org/topic/nutrition/malnutrition/ (accessed November 2018).

4. Patwari AK, Kumar S \& Beard J (2015) Undernutrition among infants less than 6 months of age: an underestimated public health problem in India. Matern Child Nutr 11(1), 119126.

5. Symonds ME (2013) Review Article: Brown Adipose Tissue Growth and Development. Scientifica. Published online: 28 February 2013. doi: http://dx.doi.org/10.1155/2013/305763.

6. Gervassi AL \& Horton H (2014) Is Infant Immunity Actively Suppressed or Immature? Virology 27(5), 1-9.

7. Wensveen FM, Valentic S, Sestan M et al. (2015) Interactions between adipose tissue and the immune system in health and malnutrition. Semin Immunol 27(5), 322-333.

8. Fabio M (2014) Nutrition for Refugee Children: Risks, Screening and Treatment. Curr Probl Pediatr Adolesc Health Care 44(7), 188-195.

9. Jackson AA (2014) Feeding the normal infant, child and adolescent. Medicine 43(2) 127 131.

10. Pemunta NV \& Fubah MA (2015) Socio-cultural determinants of infant malnutrition in Cameroon. J Biosoc Sci 47(4), 423-448.

11. Raman S, Nicholls R, Ritchie J et al. (2016) Eating soup with nails of pig: thematic synthesis of the qualitative literature on cultural practices and beliefs influencing perinatal nutrition in low and middle income countries. BMC Pregnancy Childbirth 16(1), 192.

12. World Health Organisation (WHO) (2018) Media Centre: Infant and Young Child Feeding: Fact Sheet. http://www.who.int/mediacentre/factsheets/fs342/en/ (accessed November 2018)

13. World Health Organisation (WHO) (2015) World Health Statistics 2015. Geneva: World Health Organisation. 
14. Kerac M, Frison S, Connell N et al. (2019) Informing the management of acute malnutrition in infants aged under 6 months (MAMI): risk factor analysis using nationally-representative demographic \& health survey secondary data. PeerJ 6, e5848.

15. Lopriore C, Dop MC, Solal-Céligny A et al. (2007) Excluding infants under 6 months of age from surveys: impact on prevalence of pre-school undernutrition. Public Health Nutr 10(1), 79-87.

16. Bryce J, Boschi-Pinto C, Shibuya K et al. (2005) WHO estimates of the causes of death in children. Lancet 365, 1147-1152.

17. Grijalva-Eternod CS, Kerac M, McGrath M et al. (2017) Admission profile and discharge outcomes for infants aged less than 6 months admitted to inpatient therapeutic care in 10 countries: A secondary data analysis. Matern Child Nutr 13, e12345.

18. United Nations Children's Fund (UNICEF) (2011). Programming Guide: Infant and Young Child Feeding. New York: UNICEF.

19. Food and Agriculture Organization \& World Health Organisation (FAO \& WHO) (2016) United Nations Decade of Action on Nutrition 2016-2025: Frequently Asked Questions. http://www.fao.org/3/a-i6137e.pdf (accessed March 2017).

20. Scaling Up Nutrition (SUN) (2015) The SUN Movement Strategy and Roadmap. https://scalingupnutrition.org/wpcontent/uploads/2016/09/SR_20160901_ENG_web_pages.pdf (accessed March 2017).

21. World Health Organisation (WHO) (2013). Guideline: Updates on the management of severe acute malnutrition in infants and children. Geneva: World Health Organization.

22. Ahmad UN, Yiwombe M, Chisepo P et al. (2014) Interpretation of World Health Organization growth charts for assessing infant malnutrition: A randomised controlled trial. J Paediatr Child Health 50(1), 32-39.

23. Angood C, McGrath M, Mehta S et al. (2015) Research Priorities to Improve the Management of Acute Malnutrition in Infants Aged Less Than Six Months (MAMI). PLoS Med 12(4).

24. United Nations Children's Fund (UNICEF) (2012) Nutrition Glossary. https://www.unicef.org/lac/NutritionGlossary(3).pdf (accessed March 2017).

25. Ralston ME \& Myatt MA (2016) Weight Estimation Tool for Children Aged 6 to 59 Months in Limited-Resource Settings. PLoS One 11(8).

26. Etikan I, Musa SA \& Alkassim RS (2016) Comparison of Convenience Sampling and Purposive Sampling. Am J Theor Appl Stat 5(1), 1-4. 
27. World Health Organisation - Department of Nutrition for Health and Development (2008) Training Course on Child Growth Assessment: WHO Child Growth Standard: Interpreting Growth Indicators. Geneva: World Health Organisation.

28. World Health Organisation, World Food Programme, United Nations Standing Committee on Nutrition \& United Nations Children's Fund (WHO, WFP, UNSCN \& UNICEF) (2007) A Joint Statement by the World Health Organization, the World Food Programme, the United Nations System Standing Committee on Nutrition and United Nations Children's Fund. Community-based management of severe acute malnutrition. Geneva, Rome, New York: World Health Organisation, World Food Programme, United Nations Standing Committee on Nutrition \& United Nations Children's Fund.

29. Clark SL, Muthén B, Kaprio J et al. (2013) Models and strategies for factor mixture analysis: An example concerning the structure underlying psychological disorders. Struct Equ Modeling 20(4).

30. Berlin KS, Williams NA \& Parra GR (2013) An introduction to latent variable mixture modeling (part 1): overview and cross-sectional latent class and latent profile analyses. $J$. Pediatr. Psychol 39(2), 174-187.

31. Yuan K \& Bentler P (2000) Three likelihood-based methods for mean and covariance structure analysis with non-normal missing data. Sociol Methodol 30(1), 165-200.

32. Akaike H (1987) Factor analysis and AIC. Psychometrika 52(3), 317-332.

33. Schwartz G (1978) Estimating the dimension of a model. Ann. Stat 6(2), 461-464.

34. Sclove SL (1987) Application of model-selection criteria to some problems in multivariate analysis. Psychometrika 52(3), 333-343.

35. Nylund KL, Asparouhov T \& Muthen B (2007) Deciding on the number of classes in latent class analysis and growth mixture modelling: A Monte Carlo simulation study. Struct. Equ. Model 14(4), 535-569.

36. Lo Y, Mendell N \& Rubin DB (2001) Testing the number of components in a normal mixture. Biometrika 88(3), 767-778.

37. Muthen LK \& Muthen BO (2013) MPlus Statistical Analysis with Latent Variables. 7th ed. Los Angeles: Muthen and Muthen.

38. Denscombe M (2010) The good research guide for small scale social research projects. 4th ed. Berkshire: Open University Press.

39. Kerac M, McGrath M, Grijalva-Eternod C et al. (2010) Chapter 10 - the way forward. In MAMI Project. Technical Review: Current evidence, policies, practices \& programme 
outcomes, pp. 196-199. https://www.ennonline.net/attachments/980/mami-chapter-10-theway-forward.pdf (accessed March 2017).

40. United States Agency for International Development (USAID) (2016) Community-Based Management of Acute Malnutrition: Multi-Sectoral Nutrition Strategy 2014-2025: Technical Guidance Brief. Washington: USAID.

41. Arafat Y, Islam MM, Connell N et al. (2018) Perceptions of acute malnutrition and its management in infants under 6 months of age: a qualitative study in rural Bangladesh. Clin Med Insights Pediatr 12, 1-10.

42. Mwangome M, Ngari M, Fegan G, et al. (2017) Diagnostic criteria for severe acute malnutrition among infants aged under 6 mo. Am Journal Clin Nutr 105(6), 1415-1423.

43. Mwangome MK, Fegan G, Mbunya R et al. (2012) Reliability and accuracy of anthropometry performed by community health workers among infants under 6 months in rural Kenya. Trop Med Int Health 17(5), 622-629.

44. Kramer CV \& Allen, S (2015) Malnutrition in Developing Countries. Paediatr Child Health 25(9), 422-427.

45. World Health Organisation (WHO) (2006) WHO Child Growth Standards WHO Child Growth Standards: Length/height-for-age, weight-for-age, weight-for-length, weight-forheight and body mass index-for-age: Methods and development. Geneva: World Health Organisation.

46. Kerac M, Mwangome M, McGrath M et al. (2015) Management of acute malnutrition in infants aged under 6 months (MAMI): current issues and future directions in policy and research. Food Nutr Bull 36, Suppl 1, S30-S34. 
Table 1. Prevalence of U6Ms meeting the criteria for malnourishment according to international cut-offs, the results of the factor mixture model, and as per programme admittance

\section{Malnourished Nourished}

Criteria

$$
n=(\%) \quad(\%, n=)
$$

Traditional Cut-offs

WAZ

WLZ

MUAC $<115 \mathrm{~mm}$

MUAC $<110 \mathrm{~mm}$

MAMI Programme Admittance

Factor Mixture Model
$177(5.1 \%) \quad 3273(95.0 \%)$

$133(3.9 \%) \quad 3311(96.1 \%)$

$286(8.3 \%) \quad 3158(91.7 \%)$

$129(3.7 \%) \quad 3315(96.3 \%)$

$202(9.0 \%) \quad 2039(91.0 \%)$

$196(5.7 \%) \quad 3248(94.3 \%)$ 
Accepted manuscript

Table 2. Fit indices from the factor mixture models $(\mathrm{N}=3,444)$

\begin{tabular}{lcccccc}
\hline Classes & Log Likelihood & AIC & BIC & ssaBIC & LMR-A (p) & Entropy \\
\hline 1 & -16933 & 33884 & 33939 & 33911 & -- & -- \\
$\mathbf{2}$ & $\mathbf{- 1 6 8 7 9}$ & $\mathbf{3 3 7 8 0}$ & $\mathbf{3 3 8 4 8}$ & $\mathbf{3 3 8 1 3}$ & $\mathbf{1 0 1}(\mathbf{. 0 0 2})$ & $\mathbf{. 8 0}$ \\
3 & -16870 & 33767 & 33847 & 33806 & $16(.409)$ & .85 \\
4 & -16864 & 33758 & 33850 & 33802 & $12(.248)$ & .81
\end{tabular}

Note: $\mathrm{AIC}=$ Akaike Information Criterion; $\mathrm{BIC}=$ Bayesian Information Criterion; ssaBIC = sample size adjusted BIC; LMR-A = Lo-Mendell-Rubin Adjusted Test; Best fitting model in bold. 
Accepted manuscript

Table 3. Mean, standard deviation and confidence intervals (95\%) for the two-class solution

\begin{tabular}{cllll}
\hline Class & $\mathbf{n}=$ & Mean & sd & 95\% CI \\
\hline $\begin{array}{c}\text { Nourished } \\
\text { WAZ }\end{array}$ & 3248 & -0.10 & 1.29 & $(-0.15,-0.06)$ \\
WLZ & 3248 & -0.22 & 1.53 & $(-0.27,-0.16)$ \\
$\begin{array}{c}\text { MUAC } \\
\text { score) }\end{array}$ & 3248 & $\begin{array}{l}132.29 \\
(0.08)\end{array}$ & 0.93 & $(0.05,0.11)$ \\
\hline $\begin{array}{c}\text { Malnourished } \\
\text { WAZ }\end{array}$ & 196 & -3.86 & 0.91 & $(-3.99,-3.73)$ \\
$\quad$ WLZ & 196 & -1.61 & 2.01 & $(-1.90,-1.33)$ \\
$\quad \begin{array}{l}\text { MUAC } \\
\text { (Z-score })\end{array}$ & 196 & $\begin{array}{l}114.8 \\
(-1.30)\end{array}$ & 1.16 & $(-1.46,-1.14)$ \\
\hline
\end{tabular}

Note: ${ }^{*}$ MUAC scores were transformed to z-scores for the purposes of Factor Mixture

Modelling so that all measures were equally scaled; sd $=$ standard deviation; $95 \% \mathrm{CIs}=95 \%$ confidence intervals; 
Table 4. Bivariate and multivariate associations between admittance for MAMI and international criterion guidelines for acute malnutrition.

$$
\chi^{2} / \mathrm{B} \quad \mathrm{P} \quad \text { OR }(95 \% \mathrm{CI})
$$

\section{Bivariate associations}

$\begin{array}{lccc}\text { WAZ }<-3.0 & 229.23 & <.001 & 8.26(6.06,11.26) \\ \text { WLZ }<-3.0 & 117.39 & <.001 & 5.16(3.74,7.13) \\ \text { MUAC }<115 \mathrm{~mm} & 1639.45 & <.001 & 538.28(301.15,962.14) \\ \text { Gender }(0=\text { Male, } 1=\text { Female }) & 7.55 & .006 & 1.51(1.12,2.03) \\ \quad \text { Multivariate associations } & & & \\ \text { Logistic regression model } & 957.54 & <.001 & \\ \text { WAZ }<-3.30 & .75 & .025 & 2.12(1.01,4.09) \\ \text { WLZ }<-3.0 & 1.06 & .004 & 2.89(1.41,5.92) \\ \text { MUAC }<115 \text { mm } & 5.90 & <.001 & 366.03(196.82,680.74) \\ \text { Gender }(0=\text { Male, } 1=\text { Female }) & .46 & .123 & 1.58(.88,2.84) \\ \text { Age } & -.23 & .044 & .80(.64, .99)\end{array}$

Note: $\chi^{2}=$ chi-square value; $\mathrm{B}=$ unstandardized beta value; $\mathrm{P}=$ statistical significance value; OR $(95 \% \mathrm{CI})=$ Odds ratio with $95 \%$ confidence intervals; all comparisons have 1 degree of freedom; logistic regression model has 5 degrees of freedom. 
Accepted manuscript

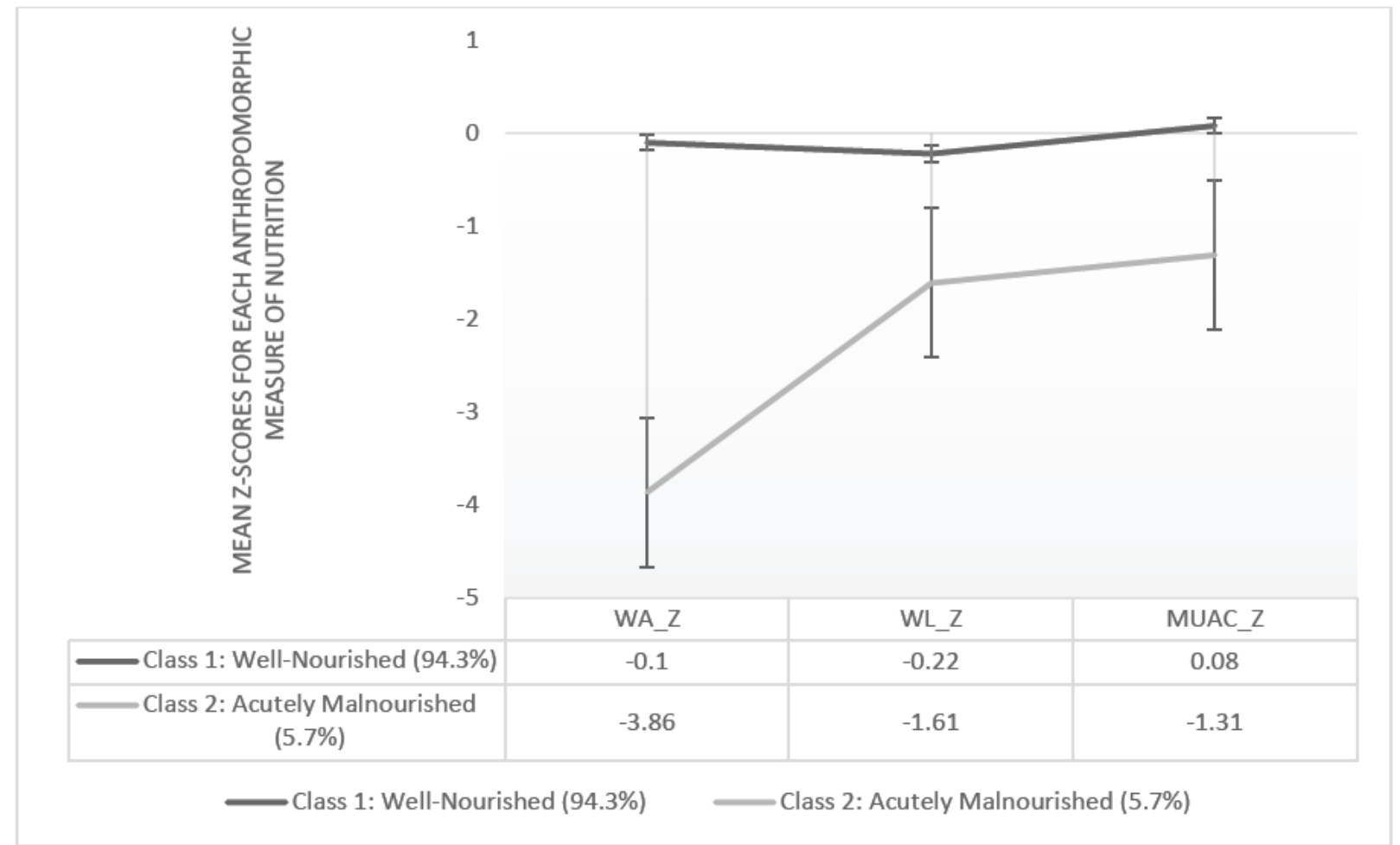

Figure 1. Mean z-scores for each anthropomorphic measure of nutrition. 